\title{
Versatile Visual Servoing without Knowledge of True Jacobian
}

\author{
Koh HOSODA and Minoru ASADA \\ Dept. of Mechanical Engineering for Computer-Controlled Machinery, Faculty of Engineering, \\ Osaka University, Suita, Osaka 565, Japan
}

\begin{abstract}
In this paper, we propose a versatile visual servoing control scheme with a Jacobian matrix estimator. The Jacobian matrix estimator does not need a priori knowledge of the kinematic structure and parameters of the robot system, such as camera and link parameters. The proposed visual servoing control scheme ensures the convergence of the image-features to desired trajectories, by using the estimated Jacobian matrix, which is proved by the Lyapunov stability theory. To show the effectiveness of the proposed scheme, simulation and experimental results are presented.
\end{abstract}

\section{Introduction}

The use of visual information is indispensable for robot systems to accomplish given tasks in dynamic environments. Many vision researchers have been adopting deliberative approaches in order to describe 3 -D scene structure, which are very time consuming, and therefore utilizing these methods to real robot applications seems hard. Recently, there have been many studies on visual servoing control schemes utilizing the visual sensors to increase the capability of the robot systems in the dynamic environments [1]. Here the robot system consists of the control scheme, kinematic structure and system parameters such as link and camera parameters. In these days, many studies focus on control schemes which make features on the image plane converge to the desired values [2-9].

Most of the previous works on visual servoing assume that the system structure and the system parameters are known, or the parameters can be identified in an off-line process (for example, $[10]$ ). A control scheme with off-line parameter identification is not robust for disturbance, change of parameters, and unknown environments. To overcome such defects, some on-line parameter identification schemes are proposed [6-9]. Weiss et al. [6] assumed that the system can be modeled by the linear and single input/ single output (SISO) equations, and applied independent MRAC(Model Reference Adaptive Control) controllers. Feddema et al. [7] and Papanikolopoulos et al. [8] modeled the system making use of ARMAX (autoregressive with external inputs) model and estimated the coefficients of the model. Papanikolopoulos et al. [9] estimated the depth related parameters. In these approaches, the structure of the system has to be known. These methods are only applicable to a restricted case, for example, a single camera case.

In this paper, we propose a versatile visual servoing control scheme with a Jacobian matrix estimator. It has the following features:

1. The Jacobian matrix estimator does not need any a priori knowledge of the kinematic structure or the system parameters, as far as all the variables of the system is measurable. So we can eliminate the exhausting calibration process.

2. There are no restrictions: number of cameras, camera in hand or camera and hand, SISO or MIMO. The proposed method is applicable to all cases.

3. The aim of the Jacobian matrix estimator is not to estimate the true parameters, but to ensure asymptotical convergence of the image-features to the desired values under the proposed control scheme. Therefore the estimated parameters do not necessarily converge to the true values.

Because of these features, the control scheme does not need to care the complexity of the system structure, the number of cameras, nor the situations of cameras (whether in-hand or fixed to the world coordinate frame). In this sense, we call the proposed scheme a versatile control scheme.

This paper is organized as follows. First, from an equation that the estimated Jacobian matrix should hold, a Jacobian matrix estimator is derived in continuous-time domain and then, modified for discrete-time domain. Another estimator is proposed based on the extended least squares algorithm, and is compared with the modified discrete-time estimator. These two kinds of estimator are unified. Then a versatile visual servoing control scheme is proposed including the feedforward term, using the estimated Jacobian matrix. The asymptotical convergence of the image-features to the time-variant desired values is proved by the Lyapunov stability theory. Finally simulation and experimental results show the effectiveness of the proposed scheme.

\section{Real-time Jacobian Matrix Estima- tor}

\subsection{Jacobian Matrix}

Only one assumption the proposed servoing control scheme needs is that variables which describe the system displacement are all measurable (See figure 1) . Using the information from sensors, we construct a 


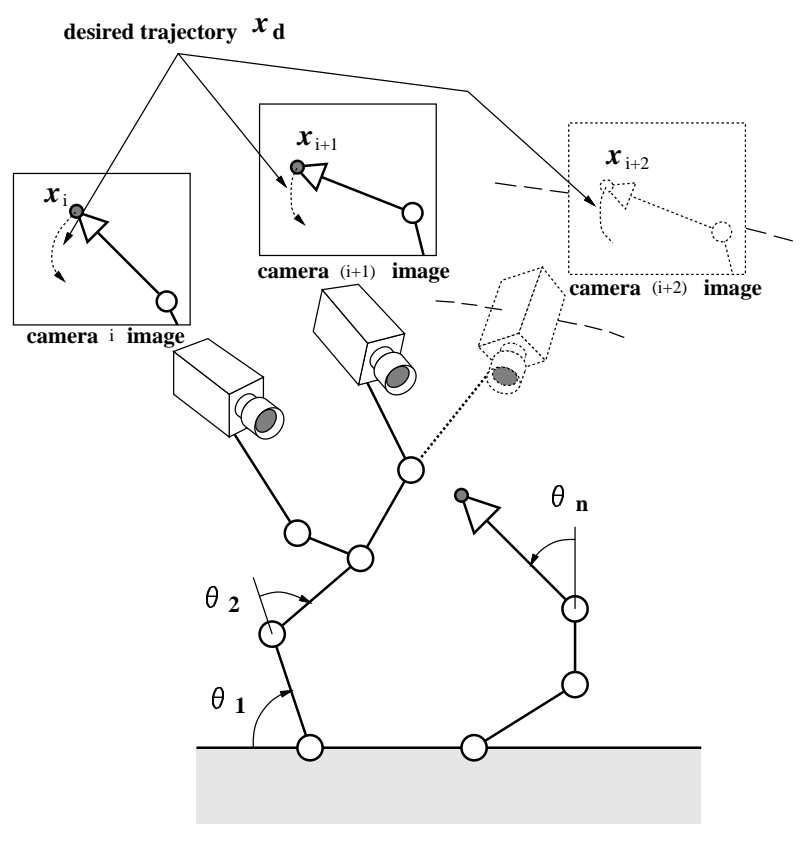

Figure 1: Robot system equipped with visual sensors

\section{Jacobian matrix estimator.}

Let $\boldsymbol{\theta} \in \Re^{n}$ and $\boldsymbol{x} \in \Re^{m}$ denote the vector consisting of variables of the system and the feature vector that is obtained from visual sensors, respectively. A relationship between $\boldsymbol{\theta}$ and $\boldsymbol{x}$ is expressed as,

$$
\boldsymbol{x}=\boldsymbol{x}(\boldsymbol{\theta}) .
$$

Differentiating eq.(1), we get the velocity relationship

$$
\dot{\boldsymbol{x}}=\boldsymbol{J}(\boldsymbol{\theta}) \dot{\boldsymbol{\theta}},
$$

where $\boldsymbol{J}(\boldsymbol{\theta})=\partial \boldsymbol{x} / \partial \boldsymbol{\theta}^{T} \in \Re^{m \times n}$ is a Jacobian matrix which denotes the relation between time-derivative of features and variables of the system. This Jacobian matrix consists of the kinematic structure, the camera parameters such as focal length, aspect ratio, distortion coefficients, and the kinematic parameters such as translation and rotation between the camera and the arm-tip and the length of links. The matrix also consists of joint variables and translation and rotation between the camera and the features.

\subsection{Jacobian Estimation Using Formal Kinematic Equation}

The Jacobian matrix is estimated to satisfy eq.(2), not to obtain the true Jacobian matrix. That is, the estimated Jacobian matrix $\widehat{\boldsymbol{J}}(t)$ is supposed to satisfy the equation

$$
\dot{\boldsymbol{x}}=\widehat{\boldsymbol{J}}(t) \dot{\boldsymbol{\theta}}
$$

Differentiating eq.(3), we get

$$
\ddot{\boldsymbol{x}}=\widehat{\boldsymbol{J}} \ddot{\boldsymbol{\theta}}+\dot{\hat{\boldsymbol{J}}} \dot{\boldsymbol{\theta}}
$$

and then

$$
\dot{\widehat{J}} \dot{\theta}=\ddot{\boldsymbol{x}}-\widehat{\boldsymbol{J}} \ddot{\boldsymbol{\theta}}
$$

The derivative $\dot{\hat{\boldsymbol{J}}}(t)$ which satisfies eq. $(5)$ cannot be determined uniquely. We choose one of the solutions of this equation:

$$
\begin{array}{ll}
\dot{\hat{\boldsymbol{J}}}=\frac{(\ddot{\boldsymbol{x}}-\widehat{\boldsymbol{J}} \ddot{\boldsymbol{\theta}}) \dot{\boldsymbol{\theta}}^{T} \boldsymbol{W}(t)}{\dot{\boldsymbol{\theta}}^{T} \boldsymbol{W}(t) \dot{\boldsymbol{\theta}}}, & (\|\dot{\boldsymbol{\theta}}\| \neq 0), \\
\dot{\widehat{\boldsymbol{J}}}=\boldsymbol{O}, & (\|\dot{\boldsymbol{\theta}}\|=0),
\end{array}
$$

where $\boldsymbol{W}(t)$ is a full rank weighting matrix. This solution can be obtained by applying a pseudo-inverse matrix of $\dot{\boldsymbol{\theta}}$ to eq.(5).

Formally discretizing eq.(5) with sampling rate $\Delta t$, we get

$$
\begin{aligned}
& \widehat{\boldsymbol{J}}(t)-\widehat{\boldsymbol{J}}(t-\Delta t)= \\
& \frac{\{\Delta \boldsymbol{x}(t)-\widehat{\boldsymbol{J}}(t-\Delta t) \Delta \boldsymbol{\theta}(t)\} \Delta \boldsymbol{\theta}(t)^{T} \boldsymbol{W}(t)}{\Delta \boldsymbol{\theta}(t)^{T} \boldsymbol{W}(t) \Delta \boldsymbol{\theta}(t)},
\end{aligned}
$$

where $\Delta \boldsymbol{\theta}(t)=\boldsymbol{\theta}(t)-\boldsymbol{\theta}(t-\Delta t)$ and $\Delta \boldsymbol{x}(t)=\boldsymbol{x}(t)-$ $\boldsymbol{x}(t-\Delta t)$.

\subsection{Modification for Discrete-Time Do- main and Extended Least Squares Al- gorithm}

On the other hand, when the system moves slowly, we can use the least squares algorithm with exponential data weighting for estimating the Jacobian matrix. From the formulation of the algorithm, we get

$$
\begin{aligned}
& \widehat{\boldsymbol{J}}(t)-\widehat{\boldsymbol{J}}(t-\Delta t)= \\
& \frac{\{\Delta \boldsymbol{x}(t)-\widehat{\boldsymbol{J}}(t-\Delta t) \Delta \boldsymbol{\theta}(t)\} \Delta \boldsymbol{\theta}(t)^{T} \boldsymbol{P}(t-\Delta t)}{\rho+\Delta \boldsymbol{\theta}(t)^{T} \boldsymbol{P}(t-\Delta t) \Delta \boldsymbol{\theta}(t)},
\end{aligned}
$$

where $\rho(0<\rho \leq 1)$ is a forgetting factor. If $\rho=1$, the new information is averaged with all past data. If $\rho<$ 1 , the old data are weighed less, and the estimator can track the slowly time-varying Jacobian matrix. Here $\boldsymbol{P}(t)$ denotes a covariance matrix:

$$
\begin{aligned}
& \boldsymbol{P}(t)= \\
& \frac{1}{\rho}\left\{\boldsymbol{P}(t-\Delta t)-\frac{\boldsymbol{P}(t-\Delta t) \Delta \boldsymbol{\theta}(t) \Delta \boldsymbol{\theta}(t)^{T} \boldsymbol{P}(t-\Delta t)}{\rho+\Delta \boldsymbol{\theta}(t)^{T} \boldsymbol{P}(t-\Delta t) \Delta \boldsymbol{\theta}(t)}\right\} .
\end{aligned}
$$

The estimator (8) is valid only when $\boldsymbol{J}$ is timeinvariant. But tuning forgetting factor $\rho$, we can apply this method when the velocity of the system is small.

Eqs.(7) and (8) appear to be similar formulations although they are introduced from different policies.

\subsection{Proposed Estimator in Discrete-Time Domain}

We propose a unified formulation of eqs.(7) and (8) in this subsection.

$$
\begin{aligned}
& \widehat{\boldsymbol{J}}(t)-\widehat{\boldsymbol{J}}(t-\Delta t)= \\
& \begin{cases}\frac{\{\Delta \boldsymbol{x}(t)-\widehat{\boldsymbol{J}}(t-\Delta t)\} \Delta \boldsymbol{\theta}(t)^{T} \boldsymbol{W}(t)}{\rho+\Delta \boldsymbol{\theta}(t)^{T} \boldsymbol{W}(t) \Delta \boldsymbol{\theta}(t)}, & (\|\Delta \boldsymbol{\theta}\| \neq 0), \\
\boldsymbol{O}, & (\|\Delta \boldsymbol{\theta}\|=0),\end{cases}
\end{aligned}
$$


where $\boldsymbol{W}(t)$ and $\rho(0 \leq \rho \leq 1)$ denote a full rank weighting matrix and a forgetting factor, respectively. Note that the forgetting factor $\rho$ can be equal to 0 .

When one adopts the covariance matrix $\boldsymbol{P}(t-\Delta t)$ as the weighting matrix $\boldsymbol{W}(t)$, and let $0<\rho \leq 1$, one can get the least squares algorithm with exponential data weighting. When one adopts $\rho=0, \widehat{\boldsymbol{J}}$ satisfies eq.(3) under the condition $\Delta t \rightarrow 0$. Using the Jacobian matrix estimator (10), if $\rho$ is nearly equal to 0 , the system becomes sensitive to observed data, and if $\rho$ is nearly equal to 1 , the system becomes insensitive and stable. When the proposed scheme is applied to the real system, one has to tune $\rho$ according to noise, sensitivity, etc.

\section{Visual Servoing Controller}

Using the estimated Jacobian matrix, we construct a visual servoing control scheme which makes features on the image plane converge to the desired values. Stability of the system depends on the relationship between $m, l$, and rank of matrix $\widehat{\boldsymbol{J}} \in \Re^{m \times n}$, where $m$ and $l$ denote the dimension of the features on image plane and the number of d.o.f. of the features in the real world. For example, tracking a finger-tip point with stereo cameras, $m=4$ and $l=3$. Note that the number of d.o.f. of the features on the image plane does not exceed that in the real world, that is, rank $\widehat{\boldsymbol{J}}$ is smaller than $l$. We assume that the system has enough degrees of freedom to control, that is $n \geq \max (m, l)$, otherwise the system cannot realize the desired motion.

Giving the desired trajectories of the features on the image plane $\boldsymbol{x}_{d}$ as $C^{1}$ class, we proposed a visual servoing control scheme

$$
\dot{\boldsymbol{\theta}}=\widehat{\boldsymbol{J}}^{+} \dot{\boldsymbol{x}}_{d}+\left(\boldsymbol{I}_{n}-\widehat{\boldsymbol{J}}^{+} \widehat{\boldsymbol{J}}\right) \boldsymbol{k}-\boldsymbol{K} \widehat{\boldsymbol{J}}^{T} \boldsymbol{e}
$$

where $\boldsymbol{A}^{+}, \boldsymbol{I}_{n}, \boldsymbol{K}, \boldsymbol{k}$ and $\boldsymbol{e}$ denote a pseudo-inverse matrix of $\boldsymbol{A}$, an $n \times n$ identity matrix, a positivedefinite gain matrix, an arbitrary vector and the error vector $\boldsymbol{e}=\boldsymbol{x}-\boldsymbol{x}_{d}$, respectively. We assume that the joints are controlled by minor velocity feedback controllers so that the inputs for the joints are desired velocity values. Applying the Jacobian matrix estimator in continuous-time domain (6), and the servoing control scheme (11), we can prove that $\boldsymbol{x}$ asymptotically converges to $\boldsymbol{x}_{d}$ by using the Lyapunov stability theory as follows;

We define a positive-definite scalar function

$$
V=\frac{1}{2} \boldsymbol{e}^{T} \boldsymbol{e}
$$

Differentiating $V$ with respect to time, we get

$$
\begin{aligned}
\dot{V} & =\boldsymbol{e}^{T} \dot{\boldsymbol{e}} \\
& =\boldsymbol{e}^{T}\left(\dot{\boldsymbol{x}}-\dot{\boldsymbol{x}}_{d}\right)
\end{aligned}
$$

Since the estimator (6) satisfies eq.(3), from eq.(11) we get

$$
\dot{V}=\boldsymbol{e}^{T}\left(\widehat{\boldsymbol{J}} \widehat{\boldsymbol{J}}^{+} \dot{\boldsymbol{x}}_{d}-\dot{\boldsymbol{x}}_{d}-\widehat{\boldsymbol{J}} \boldsymbol{K} \widehat{\boldsymbol{J}}^{T} \boldsymbol{e}\right)
$$

To show that $\dot{V}<0$ and $V$ is a Lyapunov function, we consider two cases.

(case i) $m \leq l$ : This case means that the dimension of the features on the image plane is smaller than the number of d.o.f. of the features in the real world. When $\operatorname{rank} \widehat{\boldsymbol{J}}=m$ holds, we get

$$
\widehat{\boldsymbol{J}} \widehat{\boldsymbol{J}}^{+}-\boldsymbol{I}_{m}=\boldsymbol{O},
$$

therefore the time-derivative of $V$ becomes

$$
\dot{V}=-\boldsymbol{e}^{T} \widehat{\boldsymbol{J}} \boldsymbol{K} \widehat{\boldsymbol{J}}^{T} \boldsymbol{e} \leq 0 .
$$

Here the sign of equality holds when $\boldsymbol{e}=\mathbf{o}$, therefore from the Lyapunov stability theory, the convergence $\boldsymbol{x} \rightarrow \boldsymbol{x}_{d}$ is proved as time tends to infinity.

(case ii) $m>l$ : This case means that the dimension of the features on the image plane is larger than the number of d.o.f. of the features in the real world, that is, the case that the visual information is redundant. In such a case, there is a certain constraint between the features, which means the constraint in the real world. Picking up independent elements from features, we take a new independent feature vector $\boldsymbol{y} \in \Re^{l}$. The constraint between $\boldsymbol{x}$ and $\boldsymbol{y}$ is written as

$$
\dot{\boldsymbol{x}}=\boldsymbol{J}_{i} \dot{\boldsymbol{y}},
$$

where $\boldsymbol{J}_{i} \in \Re^{m \times l}$ is a matrix which describes the constraint. Suppose that the desired trajectories $\boldsymbol{x}_{d}$ satisfies the constraint (17). When $\operatorname{rank} \widehat{\boldsymbol{J}}=l$ holds,

$$
\left(\widehat{\boldsymbol{J}} \widehat{\boldsymbol{J}}^{+}-\boldsymbol{I}_{m}\right) \boldsymbol{J}_{i}=\mathbf{o} .
$$

therefore the derivative of $V$ becomes

$$
\dot{V}=-\boldsymbol{e}^{T} \widehat{\boldsymbol{J}} \boldsymbol{K} \widehat{\boldsymbol{J}}^{T} \boldsymbol{e} \leq 0 .
$$

Here the sign of equality holds when $\boldsymbol{y}=\boldsymbol{y}_{d}$, so from the Lyapunov stability theory, the convergence $\boldsymbol{y} \rightarrow \boldsymbol{y}_{d}$ is proved as time tends to infinity. From the constraint (17), finally the asymptotical convergence $\boldsymbol{x} \rightarrow \boldsymbol{x}_{d}$ is proved.

From the cases (i) and (ii), when $\operatorname{rank} \widehat{\boldsymbol{J}}=\min (m, l)$ holds, the visual servoing control scheme (11) with the Jacobian matrix estimator (6) is proved to guarantee the asymptotical convergence $\boldsymbol{x} \rightarrow \boldsymbol{x}_{d}$. When one applies the Jacobian matrix estimator in discrete-time domain (10), the case that $\rho=0$ is proved to be stable as the sampling time $\Delta t$ tends to 0 . In the case that $\rho \neq 0$, the response of the system is supposed to be similar.

Using the proposed visual servoing control scheme with the Jacobian matrix estimator, without having any a priori knowledge of the kinematic structure and the system parameters, the features can be controlled to converge to the desired values. The typical feature of the proposed scheme is not to estimate the true parameters, but to obtain arbitrary parameters that ensure the features' asymptotical convergence to the desired values. 


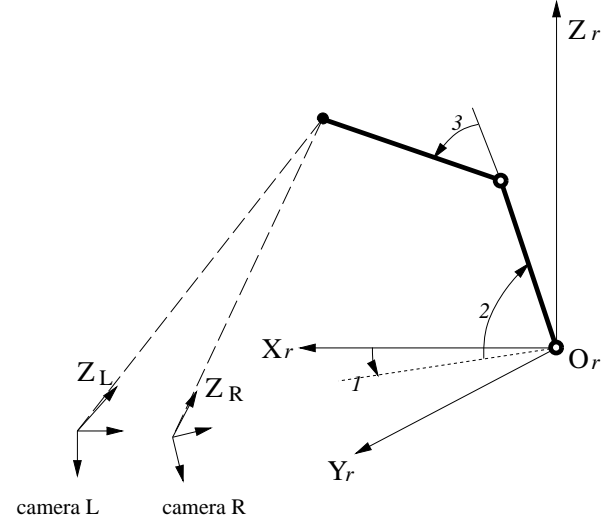

Figure 2: Robot system used for simulation

\begin{tabular}{|ll|l|}
\hline length of link 2 & $(\mathrm{m})$ & 0.43 \\
\hline length of link 3 & $(\mathrm{m})$ & 0.40 \\
\hline \hline focal length of camera R & $(\mathrm{m})$ & 0.01 \\
\hline focal length of camera L & $(\mathrm{m})$ & 0.011 \\
\hline
\end{tabular}

Table 1: Parameters of robot system

\section{Simulation}

\subsection{Robot System Used for Simulation}

To show the validity of the proposed scheme, we show some simulation results. The hardware setup of the robot system used for simulation is shown in figure 2 , which has a 3 d.o.f robot arm and two cameras. This is a model of a real robot system (figure 5). We assume a pinhole camera model as a camera model. Translation and rotation of the camera frames $\Sigma_{L}$ and $\Sigma_{R}$ with respect to the robot base frame $\Sigma_{r}$, are set:

$$
{ }^{r} \boldsymbol{R}_{R}=
$$

$$
\begin{aligned}
& {\left[\begin{array}{cccc}
-8.9 \times 10^{-1} & -3.7 \times 10^{-1} & 2.4 \times 10^{-1} & -0.1 \\
-7.3 \times 10^{-2} & -4.1 \times 10^{-1} & -9.1 \times 10^{-1} & 2.0 \\
4.4 \times 10^{-1} & -8.3 \times 10^{-1} & 3.4 \times 10^{-1} & 0.1 \\
0 & 0 & 0 & 1
\end{array}\right]} \\
& { }^{r} \boldsymbol{R}_{L}= \\
& {\left[\begin{array}{cccc}
-8.8 \times 10^{-1} & -4.5 \times 10^{-1} & 1.7 \times 10^{-1} & 0.1 \\
-1.5 \times 10^{-2} & -3.2 \times 10^{-1} & -9.5 \times 10^{-1} & 2.0 \\
4.8 \times 10^{-1} & -8.3 \times 10^{-1} & 2.8 \times 10^{-1} & 0.1 \\
0 & 0 & 0 & 1
\end{array}\right]}
\end{aligned}
$$

True parameters used for the simulation are shown in Table 1. The arm-tip point is assumed to be tracked by making use of a visual tracking system (for example, presented in [11]), then the output of the cameras is the feature vector $\left[x_{R}, y_{R}, x_{L}, y_{L}\right]^{T}$. In this simulation, video plain is 256 width $\times 480$ [pixel] height, and zero-mean noise $\pm 2[$ pixel] is added to the observed image. The joint displacement is assumed to be measured from some joint sensors.

Theoretically, in case of the gain matrix $\boldsymbol{K}$ is positive-definite, the proposed control scheme guarantees the asymptotical convergence. But in the simulation, because of the sampling ratio, too large gain leads to be unstable, so the gain matrix is chosen by trial and error:

$$
\boldsymbol{K}=\operatorname{diag}\left[6.0 \times 10^{5}, 6.0 \times 10^{5}, 6.0 \times 10^{5}\right]
$$

The initial Jacobian matrix at $t=0$ is assumed to be estimated coarsely. When $\left[\theta_{1}, \theta_{2}, \theta_{3}\right]^{T}=$ $\left[0,70^{\circ},-60^{\circ}\right]^{T}$, the estimated Jacobian matrix at $t=0$ is given as

$\widehat{\boldsymbol{J}}(0)=\left[\begin{array}{ccc}-1.0 \times 10^{-4} & 0.0 & 1.0 \times 10^{-4} \\ 0.0 & 1.0 \times 10^{-3} & -1.0 \times 10^{-3} \\ 0.0 & -1.0 \times 10^{-3} & 0.0 \\ -1.0 \times 10^{-4} & 1.0 \times 10^{-3} & -1.0 \times 10^{-3}\end{array}\right]$,

while the true Jacobian matrix is

$\boldsymbol{J}(0)=\left[\begin{array}{ccc}-7.0 \times 10^{-4} & 2.7 \times 10^{-3} & 7.0 \times 10^{-4} \\ -6.2 \times 10^{-4} & -2.2 \times 10^{-3} & -1.8 \times 10^{-3} \\ -6.3 \times 10^{-4} & 2.9 \times 10^{-3} & 5.8 \times 10^{-4} \\ -6.0 \times 10^{-4} & -2.7 \times 10^{-3} & -2.1 \times 10^{-3}\end{array}\right]$.

Sampling ratio is set as $33[\mathrm{~ms}](1 / 30[\mathrm{sec}])$.

\subsection{Step Response}

We show a result of a step response of the system in figure 3 . The initial state of the arm is $\left[\theta_{1}, \theta_{2}, \theta_{3}\right]^{T}=\left[0,70^{\circ},-60^{\circ}\right]^{T}$, the initial feature vector is $\left[x_{R}, y_{R}, x_{L}, y_{L}\right]^{T}=[67,278,168,206]^{T}$. At time $t=0$, a step desired feature vector is given: $\left[x_{R}, y_{R}, x_{L}, y_{L}\right]^{T}=[91,250,192,167]^{T}$. This desired features are calculated to satisfy the constraint in the real world.

We show results of two cases:

case 1 Using the proposed control scheme (11), with the proposed Jacobian matrix estimator (10) with $\rho=0.001$ and $\boldsymbol{W}(t)=\boldsymbol{I}_{n}$.

case 2 Using the proposed control scheme (11), without any Jacobian estimator.

In figure 3 , the error norm $\left\|\boldsymbol{x}_{R}-\boldsymbol{x}_{R d}\right\|$ on the image plane of the camera $R$ is shown. The robot can not realize the desired point stably in case 2 , while it can in case 1. Comparing the results of case 1 and case 2, we can find the effectiveness of the proposed Jacobian estimator. The result of the camera $\mathrm{L}$ is similar to this one.

\subsection{Continuous Trajectory Tracking}

We show results of continuous trajectory tracking in figure 4. The desired features are calculated so as to satisfy the constraint in the real world.

Desired features move from the initial point $\left[x_{R}, y_{R}, x_{L}, y_{L}\right]^{T}=[67,278,168,206]^{T}$, to the goal 


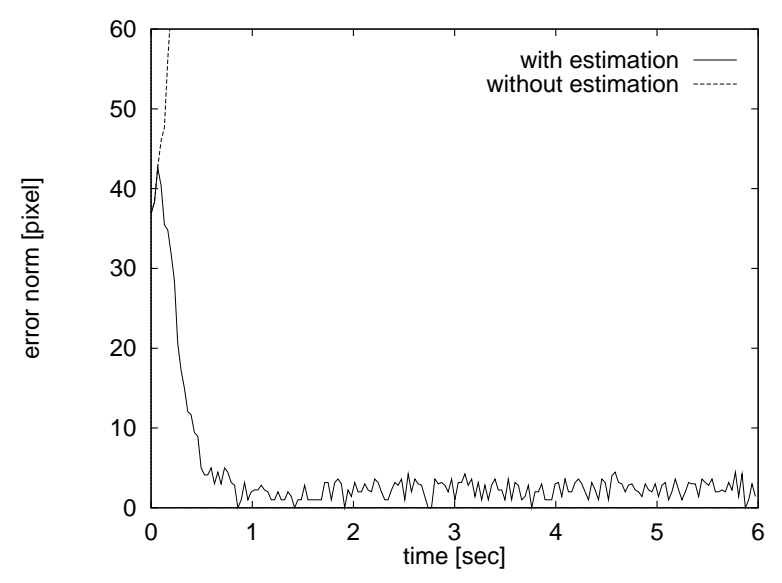

Figure 3: Simulation result (step response, error norm on camera $\mathrm{R}$ )

point $\left[x_{R}, y_{R}, x_{L}, y_{L}\right]^{T}=[65,515,169,470]^{T}$ in 3.0 [sec]. In figure 4 , we show the same cases as 1 and 2 .

The estimated Jacobian matrix at $t=6.0[\mathrm{sec}]$ in the case 1 is,

$\widehat{\boldsymbol{J}}(6)=\left[\begin{array}{ccc}-2.8 \times 10^{-3} & 1.9 \times 10^{-3} & 1.3 \times 10^{-3} \\ -1.3 \times 10^{-3} & -2.0 \times 10^{-3} & -1.4 \times 10^{-3} \\ -2.4 \times 10^{-3} & 1.7 \times 10^{-3} & -3.2 \times 10^{-3} \\ -1.3 \times 10^{-3} & -2.7 \times 10^{-3} & -6.8 \times 10^{-4}\end{array}\right]$

while the true Jacobian matrix is

$\boldsymbol{J}(6)=\left[\begin{array}{ccc}-2.1 \times 10^{-3} & 1.0 \times 10^{-3} & -2.2 \times 10^{-4} \\ -5.8 \times 10^{-4} & -3.0 \times 10^{-3} & -1.8 \times 10^{-3} \\ -2.3 \times 10^{-3} & 8.2 \times 10^{-4} & -5.4 \times 10^{-4} \\ -4.3 \times 10^{-4} & -3.4 \times 10^{-3} & -2.0 \times 10^{-3}\end{array}\right]$

Note that these two matrices are quite different, but the performances of the two cases are similar.

From these results, the proposed visual servoing control scheme with the Jacobian matrix estimator is proved effective to make features converge to desired values, without a priori knowledge of the kinematic structure and the system parameters of the robot system.

\section{$5 \quad$ Experiments}

In this section, some experimental results will be shown to demonstrate validity of the proposed scheme. In this paper, the results on PTP (Point-To-Point) control are shown. Results of two cases are shown, one of camera and arm system, and one of camera in arm system, to show the flexibility of the proposed method.

\subsection{Robot and Camera System used for experiments}

In figure 5, the experimental system used for experiments is shown. The system consists of a robot

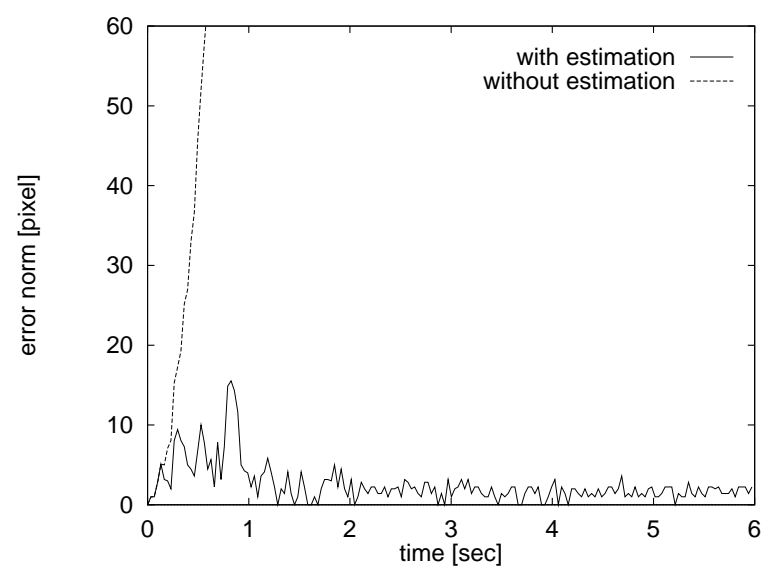

Figure 4: Simulation result (trajectory tracking, error norm no camera $\mathrm{R}$ )

manipulator and a stereo camera system. The robot manipulator is a puma type 6 d.o.f. robot manipulator, Js-5 by Kawasaki Heavy Industry. The stereo camera system consists of two color CCD cameras, ELMO UN401, whose focal length is $11[\mathrm{~mm}]$. Baseline length between two cameras is about $0.2[\mathrm{~m}]$.

\subsection{Experimental Equipment}

Figure 6 shows the experimental equipment of the system. Video signal from the stereo camera is sent to an image processing board MV200 (DataCube) and pre-processed. Then the signal is sent to the tracking module (Fujitsu). The tracking module has a function of block correlation to track some pre-memorized pattern. Then the tracking module feeds coordinates of the reference pattern to the controller, MVME167 (CPU:68040, 33MHz, motorola). The controller calculate desired position of the robot by the proposed scheme and send it to the robot controller via VMEVME bus adapter. Using the system, sampling ratio is $33[\mathrm{~ms}]$. The stereo camera system and the robot are not calibrated, so one do not know the parameters of the system completely.

\subsection{Experimental results \\ 5.3.1 Camera and arm system}

PTP control is applied to camera and arm system. The cameras are set on the ground. First we show the desired image on the image plane to the robot, and move the arm to the initial point. At the initial point, the arm moves a little in $\mathrm{x}, \mathrm{y}, \mathrm{z}$ direction, to estimate the initial coarse Jacobian matrix. Then we apply the PTP control, eq.(11) without feedforward terms. The initial image and the desired image are $\left[x_{R}, y_{R}, x_{L}, y_{L}\right]^{T}=[78,262,156,190]^{T}$ and $[106,295,185,223]$, respectively (see figure 7 ). Results of the experiment are shown in figure 8 , and figure 9 .

In figure 8 , we show two cases, with the proposed Jacobian estimation and without estimation. We can 
see the effectiveness of the proposed method from this figure.

In figure 9, we show three cases to demonstrate the effect of $\rho$. In this experiments, as $\rho$ decreases, the result becomes better. But in the real experiment, we could observe the instability as $\rho$ decreases.

\subsubsection{Camera in arm system}

To show the flexibility of the proposed scheme, the same controller is applied to a camera in arm system (figure 10). The target is fixed to the ground. We also do experiment of PTP control of this case. The result is shown in figure 11. This result demonstrates the flexibility of the proposed method.

\section{Discussion and Future Works}

In this paper, we have proposed a versatile visual servoing control scheme with a Jacobian matrix estimator to make features converge to desired values, without a priori knowledge of the kinematic structure and system parameters of the robot system. To show the validity of the proposed scheme, some simulations and real experiments are done.

When the proposed scheme is applied to the real system, we have to check how the forgetting factor $\rho$ works. The factor $\rho$ can make the system either sensitive or stable, therefore how to choose an appropriate $\rho$ is one of the future works.

We have to discuss further on the combination of the number of d.o.f. of the features in the real world $l$ and the dimension of the features in image plane $m$, which is essential for the stability of the system. Also we have to develop a method to generate the desired image features $\boldsymbol{x}_{d}$ which satisfy the real world constraint amang them.

Also to do experiments on continuous control is one of future works.

\section{References}

[1] P. I. Corke. Visual control of robot manipulators - a review. In Visual Servoing, pages 1-31. World Scientific, 1993.

[2] W. Jang and Z. Bien. Feature-based visual servoing of an eye-in-hand robot with improved tracking performance. In Proc. of IEEE Int. Conf. on Robotics and Automation, pages 2254-2260, 1991.

[3] B. Nelson, N. P. Papanikolopoulos, and P. K. Khosla. Visual servoing for robotic assembly. In Visual Servoing, pages 139-164. World Scientific, 1993.

[4] N. Maru, H. Kase, et al. Manipulator control by visual servoing with the stereo vision. In Proceedings of the 1993 IEEE/RSJ International Conference on Intelligent Robots and Systems, pages 1865-1870, 1993.

[5] K. Hashimoto, T. Kimoto, T. Ebine, and H. Kimura. Manipulator control with imagebased visual servo. In Proc. of IEEE Int. Conf. on Robotics and Automation, pages 2267-2272, 1991.
[6] L. E. Weiss, A. C. Sanderson, and C. P. Neuman. Dynamic sensor-based control of robots with visual feedback. IEEE J. of Robotics and Automation, RA-3(5):404-417, 1987.

[7] J. T. Feddema and C. S. G. Lee. Adaptive image feature prediction and control for visual tracking with a hand-eye coordinated camera. IEEE Trans. on System, Man, amd Cybernetics, 20(5):11721183, 1990.

[8] N. P. Papanikolopoulos and P. K. Khosla. Adaptive robotic visual tracking: Theory and experiments. IEEE Trans. on Automatic Control, 38(3):429-445, 1993

[9] N. P. Papanikolopoulos, B. Nelson, and P. K. Khosla. Six degree-of-freedom hand/eye visual tracking with uncertain parameters. In Proc. of IEEE Int. Conf. on Robotics and Automation, pages 174-179, 1994.

[10] R. Y. Tsai and R. K. Lenz. A new technique for fully autonomous and efficient 3d robotics hand/eye calibration. IEEE Trans. on Robotic$s$ and Automation, 5(3):345-358, 1989.

[11] M. Inaba, T. Kamata, and H. Inoue. Rope handling by mobile hand-eye robots. In Proc. of Int. Conf. on Advanced Robotics, pages 121-126, 1993. 


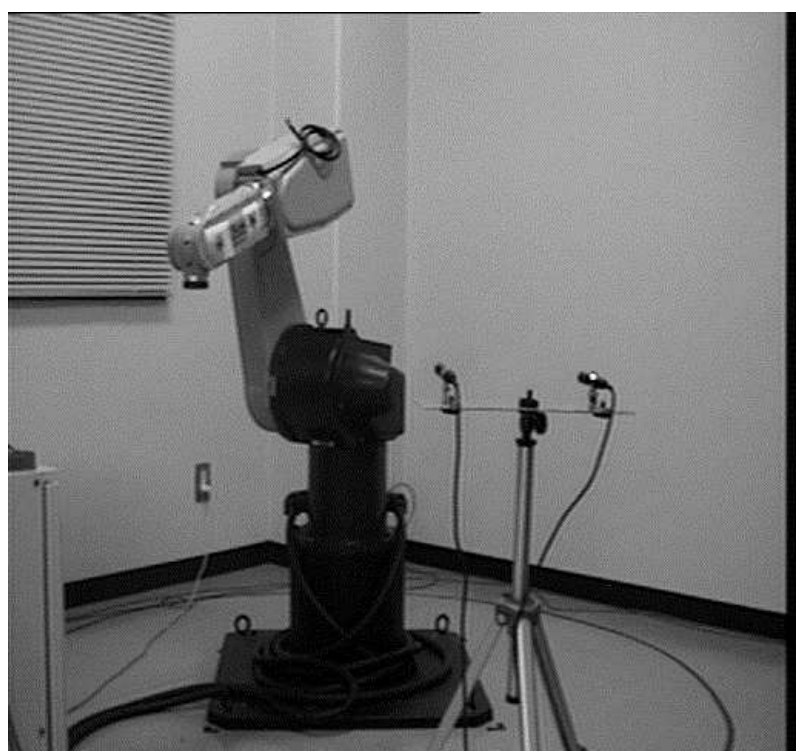

Figure 5: Camera and arm system used for experiments

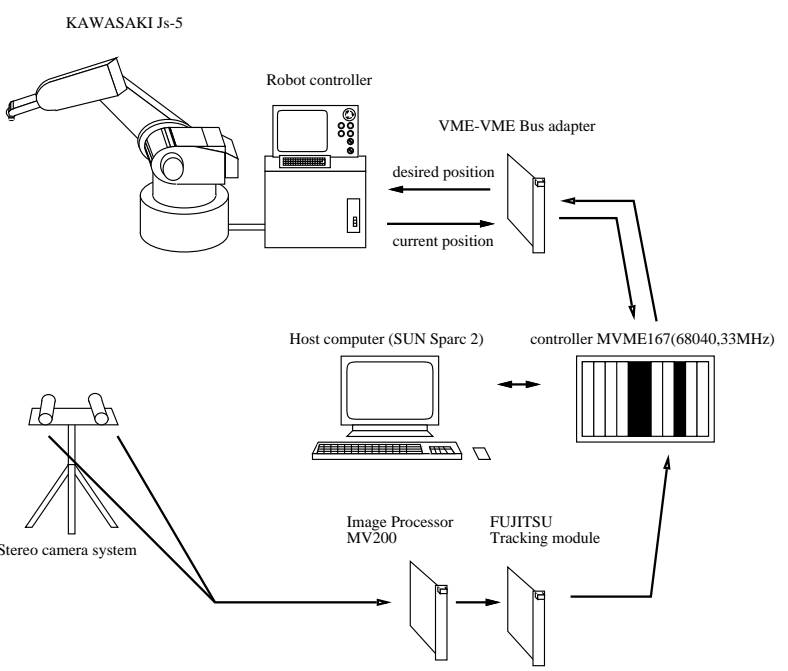

Figure 6: Experimental equipment

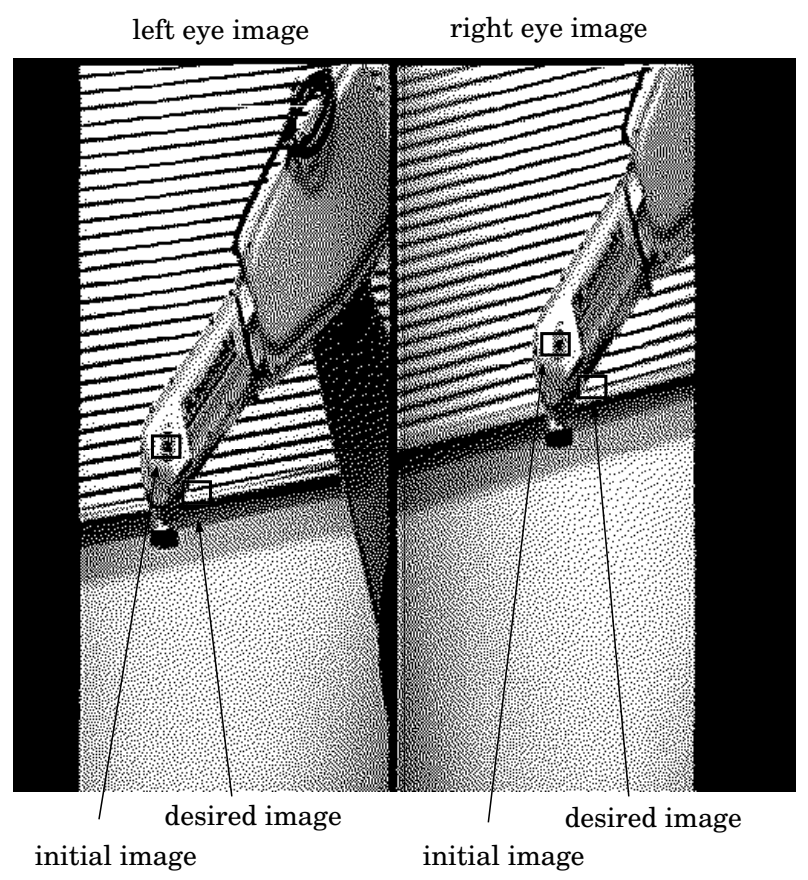

Figure 7: Initial and desired image

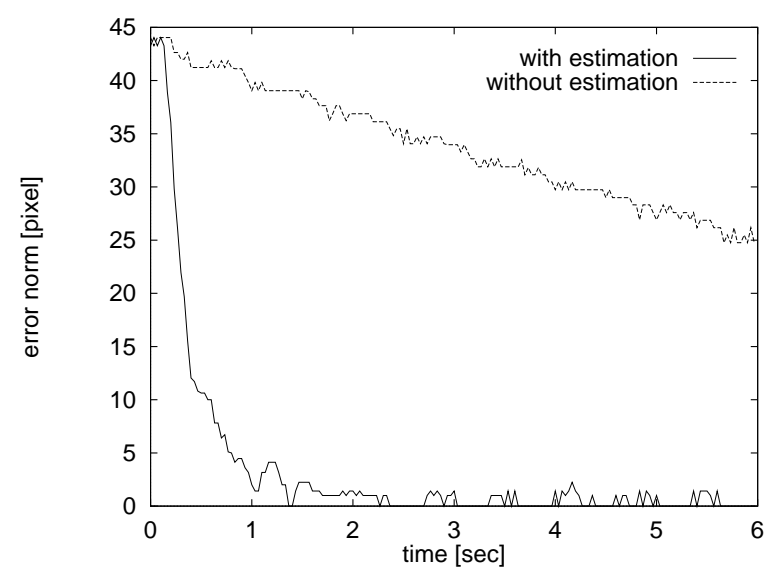

Figure 8: Experimental result 1 (camera and arm, with and without estimation) 


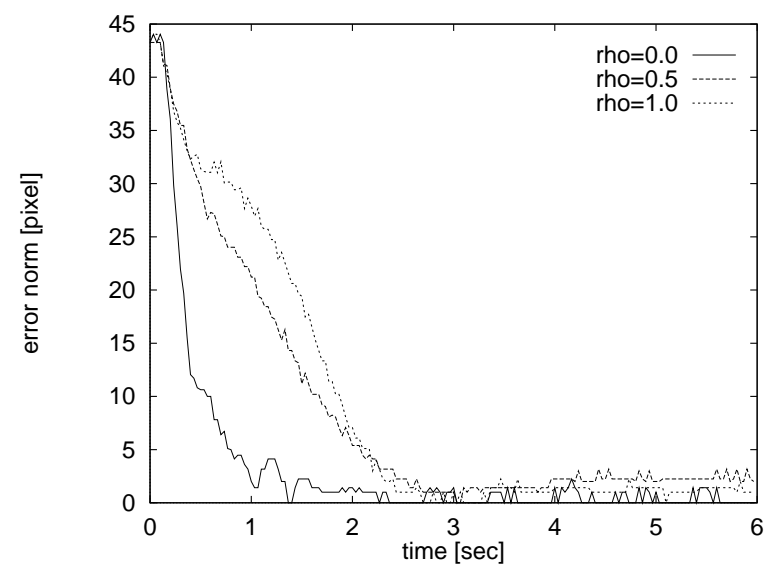

Figure 9: Experimental result 1 (camera and arm, variety of $\rho$ )

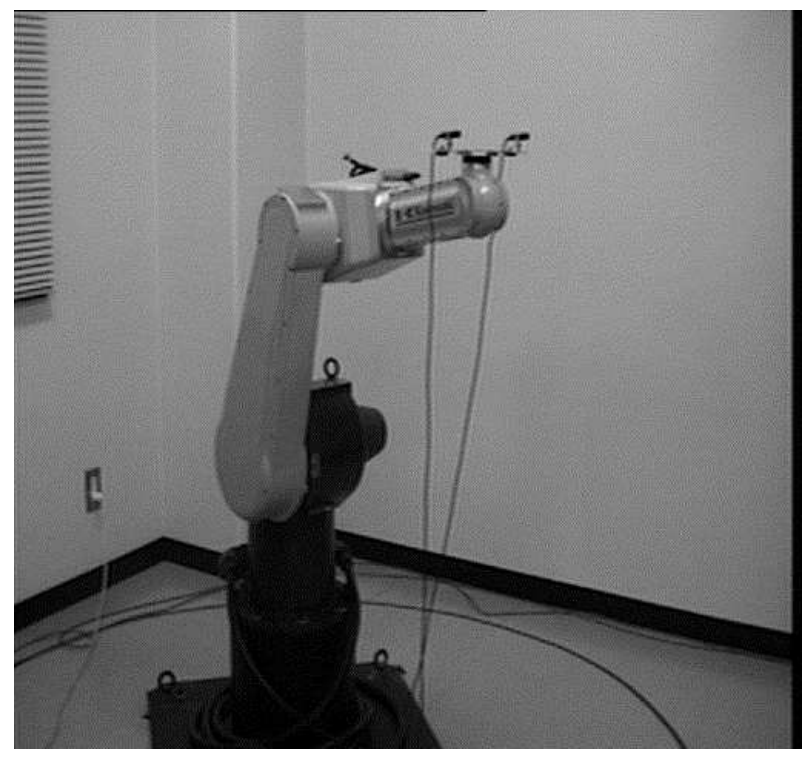

Figure 10: Camera in arm system used for experiments

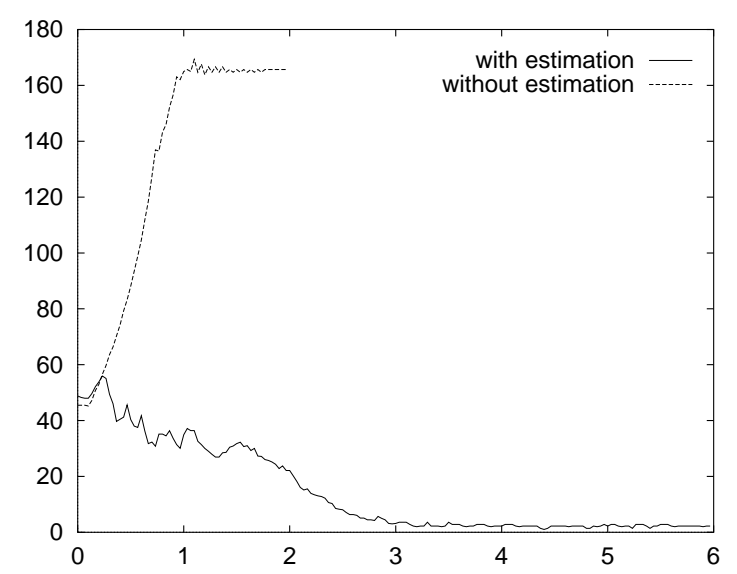

Figure 11: Experimental result 2 (camera in arm, with and without estimation) 